Neurosurg Focus 18 (1):E9, 2005

\title{
Contemporary management of incidental intracranial aneurysms
}

\author{
Ramachandra P. Tummala, M.D., Mustafa K. BaşKaya, M.D., \\ and Roberto C. Heros, M.D. \\ Department of Neurological Surgery, University of Miami School of Medicine, Miami, Florida
}

\begin{abstract}
The management of unruptured intracranial aneurysms has changed significantly in recent years and continues to evolve. The three main factors that have affected the management of unruptured intracranial aneurysms are as follows: 1) increased availability of noninvasive imaging technology, resulting in increased detection of incidental aneurysms; 2) improved understanding of the natural history of unruptured aneurysms; and 3) the advent of neuroendovascular therapy. In this report, the authors discuss the implications of these factors in the diagnosis and management of truly incidental, asymptomatic aneurysms and review the current practice patterns at their institution.

Historical and current articles regarding noninvasive neuroimaging, aneurysm screening, endovascular and surgical therapy, and the natural history of unruptured aneurysms were reviewed. Current practices used for diagnosis and management of incidental aneurysms at the authors' institution were also reviewed.

The management of incidental intracranial aneurysms has become an increasingly controversial subject in recent years. Improvements in noninvasive imaging resulting in detection of an increasing number of incidental aneurysms, the establishment of endovascular therapy as an attractive alternative to surgery, and studies indicating a more benign natural history for unruptured aneurysms than previously thought have led to significant changes in neurosurgical practice. Safety and long-term efficacy are the goals of treatment for unruptured aneurysms. Until conclusive studies are completed, the experience of the neurovascular team at each institution and the art of patient selection for treatment will continue to play a fundamental role in the management of these lesions.
\end{abstract}

\section{KEY WORDS • unruptured aneurysm • incidental intracranial aneurysm}

\section{OVERVIEW}

The management of unruptured intracranial aneurysms has changed significantly in recent years and continues to evolve. In the past, the majority of these lesions were discovered in patients with a previously ruptured aneurysm or in individuals with symptoms or signs referable to embolism or mass effect from an unruptured aneurysm. Nowadays, a larger portion of unruptured aneurysms coming to a neurosurgeon's attention are truly incidental, asymptomatic lesions detected as a result of increased use of noninvasive neuroimaging to evaluate vague symptoms such as dizziness and headaches. Or, as is becoming common practice in Japan, we have noticed that imaging is performed simply to screen for an intracranial lesion as part of a routine "brain check" examination. Presently, almost one third of intracranial aneurysms treated at our center fall into this latter category.

The three main factors that have affected the management of unruptured intracranial aneurysms are as follows: 1) increased availability of noninvasive imaging technol-

Abbreviations used in this paper: CT = computerized tomography; ISAT = International Subarachnoid Aneurysm Trial; ISUIA = International Study of Unruptured Intracranial Aneurysms; MR = magnetic resonance; $\mathrm{SAH}=$ subarachnoid hemorrhage. ogy, resulting in increased detection of incidental aneurysms; 2) improved understanding of the natural history of unruptured aneurysms; and 3) the advent of neuroendovascular therapy. Consequently, the neurosurgeon is now confronted with increasingly complex management decisions regarding the patient harboring an unruptured aneurysm. In this report, we shall discuss the implications of the aforementioned factors in the diagnosis and management of truly incidental, asymptomatic aneurysms and review the current practice patterns at our institution.

\section{Increased Detection of Unruptured Aneurysms}

Advances in noninvasive imaging modalities such as $\mathrm{CT}$ and MR angiography have markedly improved our ability to detect unruptured intracranial aneurysms. The rate of detection of aneurysms increased from 0.3 to 2 per 100,000 person-years between 1965 and 1995. ${ }^{25}$ These imaging tools are being used to screen patients with a family history of SAH, those with nonspecific neurological complaints, or as mentioned earlier, simply to rule out intracranial pathological conditions as part of a routine examination.

Computerized tomography angiography is a relatively new modality in which rapid injection of an iodinated contrast agent is followed by thin-slice imaging during the ar- 
terial phase of contrast opacification. Three-dimensional reconstruction techniques may be used to improve anatomical detail. Although CT angiography is effective in the detection of aneurysms, its specificity and sensitivity are dependent on lesion size and on institutional experience. The dense bone at the skull base limits the capabilities of CT angiography for detection of very small aneurysms..$^{33}$ The sensitivity for detecting an aneurysm larger than $5 \mathrm{~mm}$ in diameter is 95 to $100 \%$. For lesions smaller than $5 \mathrm{~mm}$, the sensitivity ranges from 64 to $83 \%$. Advances in CT angiography technology may improve sensitivity for smaller lesions. The specificity of CT angiography is between 79 and $100 \%$, and the specificity for aneurysms larger than $5 \mathrm{~mm}$ is 97 to $100 \% .^{18,31}$

The sensitivity of MR angiography varies from 55 to $100 \%$. This wide range is due to variation in aneurysm size and image reconstruction techniques. As with CT angiography, the critical aneurysm size below which the sensitivity decreases is $5 \mathrm{~mm}$. The main shortcoming of CT and MR angiography as screening tools is the reduced sensitivity for aneurysms smaller than $5 \mathrm{~mm} .{ }^{35} \mathrm{In}$ our experience, MR angiography seems to be the most common initial imaging modality used in detecting an incidental aneurysm. This is likely due to wider availability and increased familiarity with MR compared with CT angiography, and to the fact that MR imaging is frequently used first and is conveniently followed with an MR angiogram when there is a suspicion of an aneurysm. At our institution, CT angiography continues to be a supplementary study, requested mostly by neurosurgeons to aid surgical planning for complex aneurysms.

\section{Natural History of Aneurysms}

Our understanding of the natural history of unruptured aneurysms is limited but continues to evolve. In studies preceding the ISUIA, the annual risk of rupture was estimated to be 1 to $2.5 \% .{ }^{11,21,22}$ In addition, very small unruptured lesions were considered to pose a significant risk of bleeding due to potential increases in aneurysm size. ${ }^{37}$ As a result, many investigators argued for surgical treatment of essentially all detected unruptured aneurysms in young and relatively healthy patients.

The ISUIA, published in 1998, has received much attention from the neurosurgical community and the lay press. The results of this study challenged previous notions regarding the natural history of unruptured aneurysms and the correlation of lesion size to rupture risk. Because the results of the ISUIA have affected contemporary neurosurgical practice, this study deserves special mention.

The ISUIA is the largest systematic investigation to date in which the natural history of unruptured aneurysms has been evaluated. In the retrospective part of the study, researchers identified 1449 patients with 1937 unruptured aneurysms. Based on evidence that the natural history of unruptured aneurysms is different in patients with a previous SAH from a separate aneurysm compared with patients with no previous SAH, the ISUIA investigators divided the participants into two groups: patients with no history of SAH (Group 1) and those with previous SAH who had undergone treatment for the aneurysm thought to be the source of the bleeding (Group 2). The mean age of the patients in Group 1 (727 individuals) was 56 years, and $25 \%$ harbored multiple aneurysms. The mean diameter of the aneurysms was $10.9 \mathrm{~mm}$, and $58 \%$ were smaller than $10 \mathrm{~mm}$. Only $10 \%$ were located in the anterior communicating complex or anterior cerebral artery, and $16.9 \%$ were located in the cavernous sinus. In Group 2 (722 patients), the mean age was 49.4 years, and $25.2 \%$ harbored multiple lesions. Thirty-two patients among the 1449 included in the study had a documented SAH. The annual risk of SAH from an aneurysm smaller than $10 \mathrm{~mm}$ in patients in Group 1 was $0.05 \%$ per year, far less than previous observations. The rate of rupture for aneurysms larger than $10 \mathrm{~mm}$ was $1 \%$ per year. In this group, the lesion's size was the best predictor of future rupture. Also, aneurysm location was an independent risk factor for future SAH. Aneurysms located at the basilar apex, vertebrobasilar, posterior communicating, and posterior cerebral arteries had a relatively higher risk of rupture. In patients in Group 2, the annual risk of subsequent SAH from an aneurysm smaller than $10 \mathrm{~mm}$ (after treatment of the lesion thought to have been responsible for the initial bleeding) was $0.5 \%$ per year, 10 times higher than that in Group 1. Unlike in Group 1, aneurysm size did not predict the risk of future rupture in Group 2; aneurysm location at the basilar bifurcation was the only factor associated with a higher risk of future $\mathrm{SAH} .{ }^{9}$

An exhaustive review of the ISUIA is beyond the scope of this report, but several points about the study must be emphasized. The results of ISUIA have created much controversy, and the study has been criticized for possible selection bias in its retrospective cohort. ${ }^{5}$ Because each patient in the study was selected for observation by a neurosurgeon, the question immediately arises whether patients thought to have an aneurysm with a high risk of rupture were treated rather than observed and so were selected out of the study pool. If this assumption were true, then the studied population of patients would inherently be at low risk, and the results would be biased to a lower rupture rate.

An additional concern about the ISUIA is the inclusion of cavernous carotid artery aneurysms. Because the rupture of these lesions does not generally result in SAH, their inclusion in the study may have shifted the results to reflect a lower rupture rate for all aneurysms. Another concern is the discrepancy between the number of SAHs predicted by the ISUIA and the number actually observed each year. The observed annual incidence of SAH is 30 to 40 per 100,000 population, from which we can predict an aneurysm rupture rate of at least $1 \%$ per year., ${ }^{3,14,15}$ This analysis indicates that the rupture rate determined according to the findings in the ISUIA is too low.

Another fact that is difficult to reconcile with the results of this study is that a majority of aneurysms are smaller than $10 \mathrm{~mm}$ at the time of rupture. ${ }^{9}$ It has been suggested that the retrospective group had excluded patients with the highest risk of rupture and introduced data collection bias, artificially lowering the estimated annual hemorrhage rate for previously ruptured aneurysms. The ISUIA did not make a clear distinction between the bleeding risks associated with symptomatic and truly incidental unruptured aneurysms and did not determine the influence of genetic factors, smoking history, or family history. It is likely that the patients enrolled in the retrospective cohort 
between 1970 and 1991 represented only a small portion of the total group harboring unruptured, asymptomatic aneurysms seen at the 53 participating centers.

The prospective arm of the ISUIA, whose results were published last year, revealed an overall incidence of aneurysm rupture of $0.8 \%$ per year, which is much closer to our previous estimate. In this part of the study, 4060 patients from 61 centers were enrolled between 1991 and 1998. In addition to performing further assessment of the natural history of unruptured aneurysms, in this study the investigators attempted to define the risks associated with aneurysm treatment. The statistical demarcation for low rupture risk in terms of size was $7 \mathrm{~mm}$ in the new study compared with $10 \mathrm{~mm}$ in the first ISUIA. There was also a shorter mean follow-up duration of 3.9 years in the recently published ISUIA compared with 8.3 years in the first one. ${ }^{8}$ The discrepancies in rupture rates between the two arms of the ISUIA reinforce the idea that this rate may have been underestimated in the first part of the study.

The results of the ISUIA have come under additional challenge because of two recent natural history studies. In one study from Finland, 142 patients with 181 unruptured intracranial aneurysms were followed for a mean of 19.7 years. Because aneurysm surgery was not performed in Finland before 1979, all patients were observed during the study period. Of the 142 patients, 131 had suffered a previous SAH, and therefore were comparable to Group 2 of the ISUIA. The overall annual incidence of hemorrhage was found to be $1.3 \%$. Further analysis revealed that the annual rupture rate was $2.6 \%$ in symptomatic patients, $1.3 \%$ in patients with prior $\mathrm{SAH}$, and $1 \%$ in those with incidental aneurysms. ${ }^{16}$ Despite its long follow-up duration in a stable population and no inherent surgical selection bias, the major shortcomings of this study lie in its small sample size and its analysis of patients treated in the era before CT or MR imaging were available.

The second study challenging the ISUIA results was performed at a single center in Japan. In this study, 62 patients with no previous SAH in whom an unruptured aneurysm was diagnosed based on angiography were followed for at least 6 months. The risk of rupture for incidental aneurysms identified in patients with no prior SAH was reported to be much higher than previously believed. The cumulative risk of rupture for all aneurysms was $7.5 \%$ at 5 years and $22.1 \%$ at 10 years. The 5- and 10 -year cumulative SAH rates for aneurysms smaller than $10 \mathrm{~mm}$ were 4.5 and $19 \%$, respectively. ${ }^{32}$ These results were similar to those from other studies but were still higher than the ISUIA results.

Despite the criticisms and the challenges to the findings of the ISUIA, the effects of this influential study cannot be disputed. The exact rupture rates will continue to be debated, but the investigators in this study demonstrated that the natural history of unruptured aneurysms is more benign than previously thought. Guidelines based on the results of the ISUIA have been published in which observation rather than treatment is recommended for incidental aneurysms smaller than $10 \mathrm{~mm}$ in patients with no previous SAH. ${ }^{1}$ Exceptions to this are lesions approaching $10 \mathrm{~mm}$ in diameter and those with daughter sac formations and other unique hemodynamic or morphological features. Patients with a family history of aneurysmal SAH also deserve special consideration for treatment.

\section{TREATMENT OPTIONS}

\section{Endovascular Therapy}

Since its approval by the Food and Drug Administration in 1991, there has been a steady increase in coil embolization of intracranial aneurysms. Although there are serious risks associated with coil embolization, it has proved to be a relatively safe and effective modality for treating patients with both ruptured and unruptured aneurysms. Concerns regarding endovascular treatment focus on questions about its durability and the potential for aneurysm recanalization from coil compaction. The rapid pace of development in coil and microcatheter technology further complicates adequate assessment of this therapy. When coil embolization is considered for treatment, the risks associated with angiography must be weighed because this invasive procedure is still an integral part of treatment risks for unruptured aneurysms. The combined risk of permanent and transient neurological complications from angiography was $0.3 \%$ in patients with aneurysms or arteriovenous malformations who had no previous SAH. ${ }^{2}$

According to the majority of published endovascular series, complete or nearly complete aneurysm occlusion is achieved in 80 to $90 \%$ of patients. ${ }^{7,12}$ Because small aneurysm neck remnants are common after endovascular therapy, follow-up angiography is routinely performed within 6 months of completing the coil embolization. These examinations reveal some degree of aneurysm recanalization in $50 \%$ of all coil-treated aneurysms and in up to $90 \%$ of large and giant aneurysms. ${ }^{6}$ Nearly complete aneurysm occlusion with coil embolization does not necessarily prevent partial recanalization because of coil compaction. Both incomplete filling and recanalization carry a risk of aneurysm rupture, which may necessitate secondary endovascular or surgical treatment. ${ }^{24}$ As many as 15 to $20 \%$ of patients may require more than one endovascular treatment session..$^{20}$

The ISAT was a multicenter randomized endeavor in which the safety and efficacy of endovascular coil occlusion was compared with surgical clip placement in patients with ruptured aneurysms. Of the 9278 patients considered for the trial, 2143 were randomized for coil occlusion or clip placement. The other 7135 patients were excluded because it was considered that their aneurysms could be treated optimally by only one modality. Surgery was determined to be more appropriate for the majority of these excluded aneurysms, except for basilar apex lesions, most of which were treated with coils. Interim analysis of the randomized group revealed a relative risk reduction of $22.6 \%$ and an absolute risk reduction of $6.9 \%$ for dependency or death at 1 year with endovascular treatment. Moreover, during the relatively short follow-up period of the interim report, $2.6 \%$ of patients in the endovascular group suffered bleeding after treatment compared with $0.9 \%$ of patients in the surgical group. ${ }^{10}$

The ISAT is discussed in the context of unruptured aneurysms because it is tempting to extrapolate and apply the results of this study to incidental lesions. Nevertheless, the relevance of the ISAT to patients with unruptured aneurysms is only by inference, and caution should be used in applying the results derived from treatment of ruptured aneurysms when deciding how to manage incidental and/ or unruptured lesions. 


\section{Surgical Management}

An increase in lesion size on follow-up studies may warrant treatment of an unruptured aneurysm. ${ }^{15,36}$ In a study related to the Finnish report discussed earlier, a group of patients with 111 unruptured aneurysms were followed for almost 19 years. ${ }^{15}$ Subsequent rupture of these lesions was associated significantly with their growth during the follow-up period. The mean diameter of fatal ruptured aneurysms was $13.2 \mathrm{~mm}$, compared with 10 $\mathrm{mm}$ for nonfatal ruptured lesions. ${ }^{17}$

In a comparative study,,$^{13}$ investigators evaluated the outcome between coil embolization and surgical clip placement in 216 patients who were retrospectively judged in a blinded fashion to have been eligible for either surgical or endovascular treatment. Of these patients, 118 underwent surgery and 98 received coil embolization. In this study, surgery was found to be associated with higher rates of early and persistent disability and more procedure-related complications.

In a recent review, researchers reported that elective clip occlusion of asymptomatic unruptured aneurysms is associated with a morbidity rate of $10.9 \%$ and a mortality rate of $2.6 \% ; 34$ however, most of the negative results involved the treatment of large and giant aneurysms. The morbidity and mortality rates associated with surgical treatment of a subset of small anterior circulation lesions were 1.9 and $0.8 \%$, respectively. In a metaanalysis of 28 articles published between 1966 and 1992 containing data on 733 patients with unruptured aneurysms, the authors found that surgical morbidity and mortality rates were 4.1 and $1 \%$, respectively. There was no statistically significant association between morbidity or mortality rates and factors such as the year of publication, the sex or age of patients in the studies, the aneurysm size, or its location; ${ }^{19}$ however, this lack of statistical correlation may have been due to the small numbers in the subgroup of giant complex lesions and basilar aneurysms, for which surgical morbidity is higher.

In another study ${ }^{30}$ it was demonstrated that size and not location of the lesion is the main variable predicting the incidence of complications during surgery for unruptured intracranial aneurysms. In properly selected patients, aneurysms smaller than $10 \mathrm{~mm}$ can be surgically cured, with a risk of major morbidity of approximately $1 \%$. Surgical treatment of intermediate-sized aneurysms $(10-25 \mathrm{~mm}$ in diameter) results in an approximately 5\% rate of major morbidity. Conversely, aneurysms larger than $25 \mathrm{~mm}$ in diameter imply a $20 \%$ risk of significant surgical morbidity or poor outcome after surgery. In short, in unruptured aneurysms smaller than $25 \mathrm{~mm}$ in diameter, essentially $95 \%$ of patients can expect a satisfactory outcome, with cure of the aneurysm when it is surgically treated by an experienced specialist. ${ }^{30}$ Coil embolization, for which a smaller set of long-term follow-up data is available, is associated with a less than $4 \%$ morbidity rate and a $1 \%$ mortality rate. The mechanism by which incomplete coil embolization affects the aneurysm bleeding rate has not been established. According to the prospective but nonrandomized arm of the ISUIA, the combined morbidity and mortality rate for surgery at 1 year was $12.2 \%$, compared with 9.5\% for coil embolization. ${ }^{8}$

A very recent metaanalysis of surgically treated unrup- tured aneurysms included 61 studies and involved 2460 patients and at least 2568 unruptured aneurysms $(27 \%>$ $25 \mathrm{~mm}$ and $30 \%$ located in the posterior circulation). This study revealed a $2.6 \%$ mortality rate, and permanent morbidity occurred in $10.9 \%$ of patients. ${ }^{29}$

A large study ${ }^{27}$ included 1558 patients with intracranial aneurysms treated by surgical clip occlusion; $20 \%$ of these patients harbored unruptured aneurysms. Of these lesions, in $95 \%$ the outcome was favorable, and the mortality rate in this group was $0.3 \%$. An excellent outcome was more common in patients with aneurysms of the anterior circulation than in those with lesions of the posterior circulation.

\section{CURRENT PRACTICE PATTERNS}

The number of patients with incidental aneurysms referred to our institution has been rising steadily in recent years. This is most likely attributable to the increased number of noninvasive imaging studies performed to evaluate unrelated neurological symptoms or signs. The combined effect of increased incidence of unruptured aneurysms and increased endovascular therapy for ruptured aneurysms has resulted in a shift in our surgical practice. We are proportionately operating on more unruptured aneurysms and fewer ruptured ones. In rough numbers, of the approximately 150 patients with aneurysms whom we are currently treating each year, 90 to 100 present with $\mathrm{SAH}$. Half of the patients with ruptured aneurysms are treated surgically, and the other half endovascularly. The remaining 50 to 60 patients harbor unruptured aneurysms, and almost $95 \%$ of these patients are treated surgically. The exceptions are basilar apex aneurysms, most of which are treated with coil embolization. Durability of treatment is most germane to the discussion of unruptured aneurysms, especially in young patients with a long life expectancy.

We are very careful to differentiate unruptured symptomatic aneurysms from those that are truly incidental. With rare exceptions, we recommend treatment of most unruptured symptomatic aneurysms. Unruptured aneurysms become symptomatic most frequently from mass effect on the brain, cranial nerve compression, or as a result of what is probably a "small leak." Uncommonly, these lesions present with ischemic symptoms from arterial branch occlusion or emboli from a clot within the aneurysm. Relative contraindications to the surgical management of symptomatic, unruptured aneurysms include a patient's advanced age, major medical comorbidities, or intracavernous aneurysms causing mild symptoms. There are also situations in which the patient's presentation is relatively benign and the risks of treating the aneurysm appear to outweigh the benefits, particularly in older patients; in these cases, a conservative course of action may be preferable.

Whether to treat a truly incidental aneurysm remains controversial. There is no question that the ISUIA has influenced our practice, and we have become more conservative since learning of the study's results. We individualize management decisions for each patient with an incidental aneurysm. Aneurysm size and patient age are clearly the two most important factors in our decision making; because the vast majority of ruptured aneurysms 
are smaller than $10 \mathrm{~mm}$, we consider smaller lesions for treatment. We do not have age cutoffs with regard to offering treatment, but we do consider life expectancy in estimating the benefit of treatment. In general, the potential benefit seems to be outweighed by the risk associated with treatment if the remaining life expectancy of the patient is less than 20 years. ${ }^{26}$ In this context, "young" and "old" are arbitrary terms, once again reinforcing the individualization of treatment.

Although there is no definitive protocol in our institution, we recommend treatment of incidental aneurysms that are larger than 6 to $7 \mathrm{~mm}$ in diameter in relatively young ( $<65$ years), healthy patients. If the patient is very young and/or has a family history of aneurysms, or has suffered an SAH from another aneurysm, we generally recommend treatment even for lesions of a significantly smaller size. When the anticipated morbidity related to surgical treatment is very low (for example, anterior circulation aneurysms with favorable morphological features), we lower our size threshold for recommending surgery. As a result, we occasionally clip aneurysms that are only 4 to $6 \mathrm{~mm}$ in diameter. For larger aneurysms, we expand the indications for treatment and we treat patients who are older but still in relatively good medical health, or younger patients with minor comorbidities.

The decision making becomes more complex with very large or even giant aneurysms that are truly incidental, but in which the risk related to treatment is very high. In these cases age is very influential, and we tend to accept the surgical risk in a younger patient, but treat an older patient with observation and periodic imaging. If observation is chosen over treatment, patients are followed periodically with various imaging modalities. If we find an increase in aneurysm size during the follow-up period, we tend to pursue a more aggressive approach and offer surgical treatment of these lesions. Once a decision is made to treat an incidental aneurysm, the appropriate modality must be chosen.

The main goals in a patient with SAH are the prevention of aneurysm rerupture, prevention and treatment of secondary ischemic injury from vasospasm, and management of systemic complications. There is little doubt that coil embolization effectively prevents early rehemorrhage. Areas of edematous, friable brain caused by aneurysmal SAH may be further injured during surgery. The results of the ISAT likely reflect this fact and have led us to treat most patients with poor-grade SAH with coil embolization.

Because such problems are uncommon in patients with unruptured aneurysms, we prefer open microsurgery under equal conditions in these individuals. Safety and early efficacy have been demonstrated with coil embolization. Nevertheless, durability of treatment is an additional factor to consider during the management of any asymptomatic lesion. Our long experience with microsurgical clip placement clearly indicates that once an aneurysm is thus treated, it is extremely unlikely, although not impossible, for it to bleed in the future. Nevertheless, we must admit that long-term angiographic follow-up review generally has not been as rigorous in surgically treated patients as we currently expect it to be in patients treated endovascularly.
In this respect, our usual practice has been not to recommend long-term angiographic follow up in the vast majority of patients whose aneurysms appear to have been completely occluded with clips either on immediate postoperative angiography or based on direct observation during microsurgery. We admit to the suboptimal practice of not routinely performing immediate postoperative angiography in patients with relatively straightforward aneurysms that appear to be completely occluded with clips based on direct observation. Generally, we recommend long-term angiographic follow-up review only in very young patients with a psychological profile of minimal anxiety and in patients with a family history of aneurysms. We certainly could be criticized for this policy because even in the most experienced hands, there is a very small but real risk of approximately $1.5 \%$ for aneurysm regrowth after complete clip occlusion. Furthermore, the incidence of de novo aneurysm development is approximately $1.8 \%$, especially in younger patients with multiple lesions. ${ }^{4}$

Our practice of avoiding follow-up angiography after an apparently complete clip occlusion is a product of balancing the very small risk of regrowth and hemorrhage of the treated aneurysm against the adverse psychological effects on the patient who is aware of these risks. This is an example in which the art of medical decision making plays a role in weighing these risks against the adverse effects of the knowledge that an aneurysm may regrow or bleed and the prospect of serial angiography on the patient's quality of life. In short, the patient in this situation must face the reality that surgery may not have "cured" the aneurysm.

To reiterate, we generally recommend microsurgical clip placement for most incidental, sizable ( $>6-7 \mathrm{~mm})$ aneurysms of the anterior circulation as well as for lesions of the vertebral, posterior cerebral, and posterior and anterior inferior cerebellar arteries. We also recommend open surgical treatment rather than endovascular methods for most basilar aneurysms at the origin of the superior cerebellar and the posterior cerebral artery. Our surgical results with other basilar aneurysms, particularly at the basilar bifurcation, however, have not been favorable compared with the results of endovascular treatment; therefore, we currently recommend endovascular treatment for most basilar apex, basilar origin, and basilar trunk aneurysms, ruptured or not.

A point that seems intuitive but must be emphasized is that the evaluation of "risk versus benefit" is not limited to the pretreatment period. It is a process that continues into the treatment phase, until the aneurysm is secured by clip placement or coil occlusion. This must be clearly kept in mind when treating incidental aneurysms, in which the risk of hemorrhage is generally less than $1 \%$ per year. When we perform surgery or endovascular therapy for an unruptured aneurysm, we do so with the presumption that the risk the treatment entails compares favorably with the natural history of the lesion. If we face unexpected intraoperative circumstances that clearly increase the risk associated with surgery, we do not hesitate to abort the procedure. For example, we occasionally encounter a heavily calcified aneurysm neck that cannot be treated safely with clip placement (Fig. 1), or we find perforating branches 

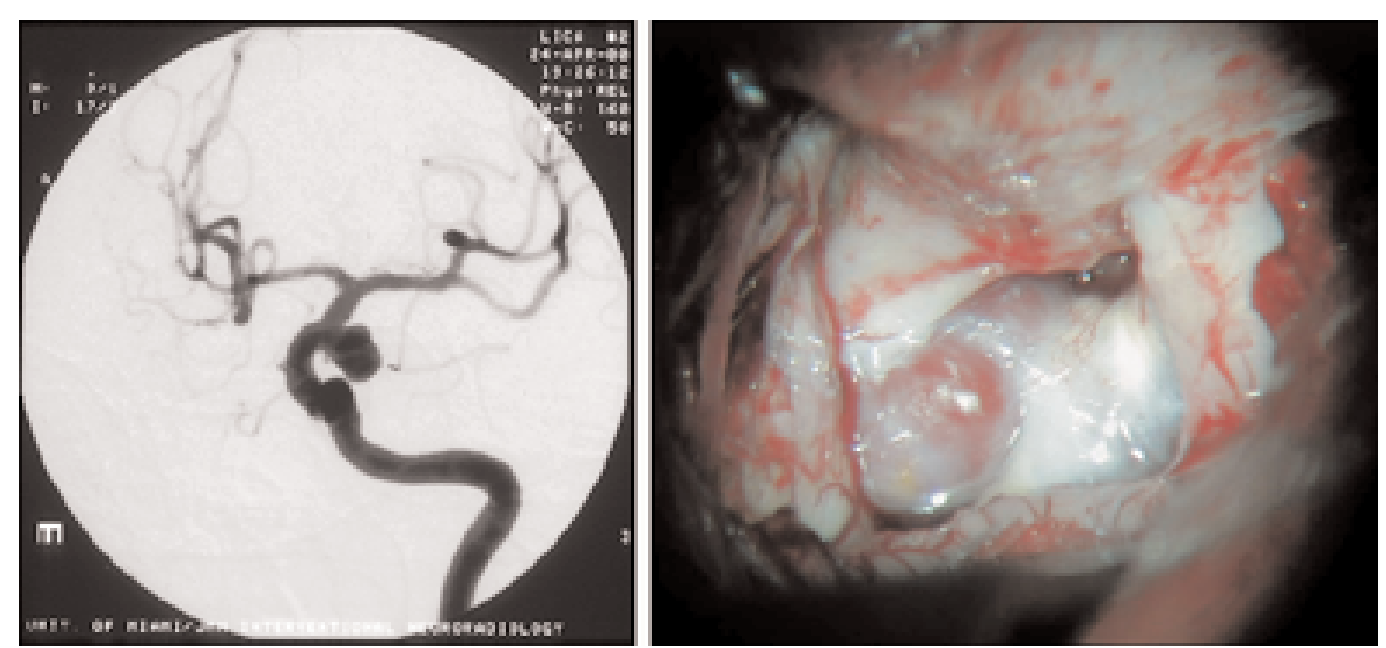

Fig. 1. Preoperative angiogram (left) revealing an 8-mm posterior communicating artery aneurysm that was discovered in a 57-year-old asymptomatic woman. Preoperatively, we estimated the risk associated with clip placement to be minimal. At surgery, a calcified atheroma that would have significantly increased the risk associated with clip placement was discovered at the neck of the aneurysm (right). We aborted the attempt at clip occlusion, and the aneurysm subsequently was treated successfully with coil embolization.

that must be sacrificed to achieve a satisfactory result. In these cases, we feel comfortable with discontinuing the procedure and either treating the patient conservatively or recommending endovascular therapy. We have the same attitude with regard to endovascular therapy, and expect our colleagues in this field to abort an operation whenever they encounter difficulties that would significantly increase the risk of the coil insertion procedure. Again, these patients may then be treated conservatively or with open microsurgery.

A topic related to unruptured aneurysms that we should discuss briefly is familial lesions. In this context, familial disease status is defined as two or more first-degree relatives with a history of SAH or an unruptured aneurysm. The available evidence indicates that screening of family members is unwarranted in cases of sporadic SAH. ${ }^{23} \mathrm{On}$ the other hand, there is evidence that screening of first-degree relatives will yield an aneurysm in $8 \%$ of persons with a history of familial SAH. ${ }^{28}$ If a history of familial aneurysms is found, we generally recommend noninvasive screening, usually by MR angiography, of first-degree relatives who are in their late teens and young or middle adulthood. We tend not to screen children because of the rare incidence of aneurysms and the difficulties in performing even noninvasive studies in this group. We also do not screen older relatives ( $>65$ years old) because the risk associated with potential treatment of an incidental aneurysm does not merit the cost of screening.

\section{CONCLUSIONS}

Management of incidental intracranial aneurysms has become an increasingly controversial subject in recent years. Improvements in noninvasive imaging resulting in detection of a rising number of incidental aneurysms, the establishment of endovascular therapy as an attractive alternative to surgery, and studies indicating a more benign natural history than previously believed for unruptured aneurysms have prompted significant changes in neurosurgical practice. Nevertheless, these advances have led to further questions and controversies. Safety and longterm efficacy are the goals of treatment for unruptured aneurysms. Until conclusive studies can be produced, the experience of the neurovascular team at each institution and the art of patient selection for treatment will continue to play a fundamental role in the management of these lesions.

\section{References}

1. Bederson JB, Awad IA, Webers DO, et al: Recommendations for the management of patients with unruptured intracranial aneurysms: a statement for healthcare professionals from the Stroke Council of the American Heart Association. Circulation 102:2300-2308, 2000

2. Cloft HJ, Joseph GJ, Dion JE: Risk of cerebral angiography in patients with subarachnoid hemorrhage, cerebral aneurysm, and arteriovenous malformation: a meta-analysis. Stroke 30: 317-320, 1999

3. Connolly ES Jr, Solomon RA: Management of unruptured aneurysms, in Le Roux PD, Winn HR, Newell DW (eds): Management of Cerebral Aneurysms. Philadelphia: Saunders, 2004, pp 271-285

4. David CA, Vishteh AG, Spetzler RF, et al: Late angiographic follow-up review of surgically treated aneurysms. J Neurosurg 91:396-401, 1999

5. Dumont AS, Lanzino G, Kassell NF: Unruptured aneurysms. J Neurosurg 96:52-60, 2002

6. Hayakawa M, Murayama Y, Duckwiler GR, et al: Natural history of the neck remnant of a cerebral aneurysm treated with the Guglielmi detachable coil system. J Neurosurg 93:561-568, 2000

7. Henkes H, Fischer S, Weber W, et al: Endovascular coil occlusion of 1811 intracranial aneurysms: early angiographic and clinical results. Neurosurgery 54:268-285, 2004

8. International Study of Unruptured Intracranial Aneurysm Investigators: Unruptured intracranial aneurysms: natural history, clinical outcome, and risks of surgical and endovascular treatment. Lancet 362:103-110, 2003 


\section{Contemporary management of incidental intracranial aneurysms}

9. The International Study of Unruptured Intracranial Aneurysm Investigators: Unruptured intracranial aneurysms - risk of rupture and risks of surgical intervention. N Engl J Med 339: 1725-1733, 1998

10. The International Subarachnoid Aneurysm Trial (ISAT) Collaborative Group: International Subarachnoid Aneurysm Trial (ISAT) of neurosurgical clipping versus endovascular coiling in 2143 patients with ruptured intracranial aneurysms: a randomised trial. Lancet 360:1267-1274, 2002

11. Jane JA, Winn HR, Richardson AE: The natural history of intracranial aneurysms: rebleeding rates during the acute and long term period and implication for surgical management. Clin Neurosurg 24:176-184, 1977

12. Johnston SC, Higashida RT, Barrow DL, et al: Recommendations for the endovascular treatment of intracranial aneurysms: a statement for healthcare professionals from the Committee on Cerebrovascular Imaging of the American Heart Association Council on Cardiovascular Radiology. Stroke 33:2536-2544, 2002

13. Johnston SC, Wilson CB, Halbach VV, et al: Endovascular and surgical treatment of unruptured cerebral aneurysms: comparison of risks. Ann Neurol 48:11-19, 2000

14. Juvela S: Treatment options of unruptured intracranial aneurysms. Stroke 35:372-374, 2004

15. Juvela S, Porras M, Heiskanen O: Natural history of unruptured intracranial aneurysms: a long-term follow-up study. J Neurosurg 79:174-182, 1993

16. Juvela S, Porras M, Poussa K: Natural history of unruptured intracranial aneurysms: probability of and risk factors for aneurysm rupture. J Neurosurg 93:379-387, 2000

17. Juvela S, Poussa K, Porras M: Factors affecting formation and growth of intracranial aneurysms: a long-term follow-up study. Stroke 32:485-491, 2001

18. Kangasniemi M, Makela T, Koskinen S, et al: Detection of intracranial aneurysms with two-dimensional and three-dimensional multislice helical computed tomographic angiography. Neurosurgery 54:336-341, 2004

19. King JT Jr, Berlin JA, Flamm ES: Morbidity and mortality from elective surgery for asymptomatic, unruptured, intracranial aneurysms: a meta-analysis. J Neurosurg 81:837-842, 1994

20. Kuether TA, Nesbit GM, Barnwell SL: Clinical and angiographic outcomes, with treatment data, for patients with cerebral aneurysms treated with Guglielmi detachable coils: a single-center experience. Neurosurgery 43:1016-1025, 1998

21. Locksley HB: Natural history of subarachnoid hemorrhage, intracranial aneurysms, and arteriovenous malformations. J Neurosurg 25:321-368, 1966

22. Locksley HB: Natural history of subarachnoid hemorrhage, intracranial aneurysms, and arteriovenous malformations. Based on 6368 cases in the cooperative study. J Neurosurg 25: 219-239, 1966

23. The Magnetic Resonance Angiography in Relatives of Patients with Subarachnoid Hemorrhage Study Group: Risks and bene- fits of screening for intracranial aneurysms in first-degree relatives of patients with sporadic subarachnoid hemorrhage. $\mathbf{N}$ Engl J Med 341:1344-1350, 1999

24. Malisch TW, Guglielmi G, Viñuela F, et al: Intracranial aneurysms treated with the Guglielmi detachable coil: midterm clinical results in a consecutive series of 100 patients. J Neurosurg 87:176-183, 1997

25. Menghini VV, Brown RD Jr, Sicks JD, et al: Incidence and prevalence of intracranial aneurysms and hemorrhage in Olmsted County, Minnesota, 1965 to 1995 . Neurology 51: 405-411, 1998

26. Mitchell P, Gholkar A, Vindlacheruvu RR, et al: Unruptured intracranial aneurysms: benign curiosity or ticking bomb? Lancet Neurol 3:85-92, 2004

27. Orz YI, Hongo K, Tanaka Y, et al: Risks of surgery for patients with unruptured intracranial aneurysms. Surg Neurol 53: 21-29, 2000

28. Raaymakers TW, Rinkel GJ, Ramos LM: Initial and follow-up screening for aneurysms in families with familial subarachnoid hemorrhage. Neurology 51:1125-1130, 1998

29. Raaymakers TWM, Rinkel GJE, Limburg M, et al: Mortality and morbidity of surgery for unruptured intracranial aneurysms: a meta-analysis. Stroke 29:1531-1538, 1998

30. Solomon RA, Fink ME, Pile-Spellman J: Surgical management of unruptured intracranial aneurysms. J Neurosurg 80: 440-446, 1994

31. Tampieri D, Leblanc R, Oleszek J, et al: Three-dimensional computed tomographic angiography of cerebral aneurysms. Neurosurgery 36:749-755, 1995

32. Tsutsumi K, Ueki K, Morita A, et al: Risk of rupture from incidental cerebral aneurysms. J Neurosurg 93:550-553, 2000

33. van Gelder JM: Computed tomographic angiography for detecting cerebral aneurysms: implications of aneurysm size distribution for the sensitivity, specificity, and likelihood ratios. Neurosurgery 53:597-606, 2003

34. Wardlaw JM, White PM: The detection and management of unruptured intracranial aneurysms. Brain 123:205-221, 2000

35. White PM, Wardlaw JM: Unruptured intracranial aneurysms. J Neuroradiol 30:336-350, 2003

36. Yasui N, Magarisawa S, Suzuki A, et al: Subarachnoid hemorrhage caused by previously diagnosed, previously unruptured intracranial aneurysms: a retrospective analysis of 25 cases. Neurosurgery 39:1096-1101, 1996

37. Yasui N, Suzuki A, Nishimura H, et al: Long-term follow-up study of unruptured intracranial aneurysms. Neurosurgery 40: 1155-1160, 1997

Manuscript received November 4, 2004.

Accepted in final form November 29, 2004.

Address reprint requests to: Roberto C. Heros, M.D., Department of Neurological Surgery, University of Miami, Lois Pope LIFE Center, 2nd Floor, 1095 NW 14th Terrace (D4-6), Miami, Florida 33136. email: rheros@med.miami.edu. 